\title{
Porous metal produced by selective laser melting with effective isotropic thermal conductivity close to the Hashin-Shtrikman bound
}

\author{
Akihiro Takezawa ${ }^{\mathrm{a}, *}$, Makoto Kobashi ${ }^{\mathrm{b}}$, Yuichiro Koizumi ${ }^{\mathrm{c}}$, Mitsuru \\ Kitamura $^{\mathrm{a}}$ \\ ${ }^{a}$ Division of Mechanical Systems and Applied Mechanics, Institute of Engineering, \\ Hiroshima University, 1-4-1 Kagamiyama, Higashi-Hiroshima, Hiroshima 739-8527, \\ Japan \\ ${ }^{b}$ Department of Materials Engineering, Graduate School of Engineering, Nagoya \\ University, Furo-cho, Chikusa-ku, Nagoya 464-8603, Japan \\ ${ }^{c}$ Institute for Materials Research, Tohoku University, 2-1-1 Katahira, Aoba-ku, Sendai \\ 980-8577, Japan
}

\begin{abstract}
Additive manufacturing may be a novel method for fabricating porous materials. These materials can achieve effective performance because of their internal geometries. Metal-additive manufacturing is expected to utilize thermal conduction materials and devices. We have developed a porous metal with effective isotropic thermal conductivity by using metal-selective laser melting additive manufacturing. The internal pore structure was designed by topology optimization, which is the most effective structural optimization technique to maximize effective thermal conductivity. The designed structure was converted to a three-dimensional STL model, which is a native digital
\end{abstract}

\footnotetext{
${ }^{*}$ Corresponding author. Tel: +81-82-424-7544; Fax: +81-82-422-7194

Email addresses: akihiro@hiroshima-u.ac.jp (Akihiro Takezawa), kobashi@numse.nagoya-u.ac.jp (Makoto Kobashi), koizumi@imr.tohoku.ac.jp (Yuichiro Koizumi), kitamura@hiroshima-u.ac.jp (Mitsuru Kitamura) 
format of additive manufacturing, and assembled as a test piece. Effective thermal conductivity was measured by a steady-state method in which the effective thermal conductivity was calculated from a one-dimensional temperature gradient and the heat flux of the test pieces. The test pieces showed an effective thermal conductivity close to the Hashin-Shtrikman or MaxwellEucken bound, which is the theoretical limit of effective performance with an error less than $10 \%$.

Keywords: Topology optimization, Porous metal, Additive manufacturing, Thermal conduction, Hashin-Shtrikman bound, Selective laser melting

\section{Introduction}

One of the research fields in the development of new materials includes developing a way to achieve macroscopic effective performance of porous or composite materials through their internal geometry. Output from this field could be used to develop various materials such as high-performance lightweight materials $[1,2,3]$, multi-functional materials $[4,5,6]$, and even special materials with extraordinarily effective physical properties such as an negative Poisson's ratio $[7,8]$ and a negative thermal expansion coefficient $[9,10]$. In addition to design based on mechanical theory $[7,1,9]$, numerical optimization could be used for the design of microstructural shapes $[10,5]$. Topology optimization, which enables fundamental optimization of structures, including changes in topology (number of holes) [11, 12], contributes to the design of materials with extreme effective physical properties $[10,8,13,5,6,14,15,16]$.

The bound for effective physical properties of internal geometry-based 
materials can be derived theoretically. Hashin-Shtrikman (HS) or MaxwellEucken bounds are representative for isotropic bulk modulus and conductivity, which is derived based on variational principle [17, 18, 19]. Finding an internal geometry design with effective properties close to such bounds could be one of the optimization goals.

Although research into optimal internal geometry design methodology has been very active for a long time, experimental verification of the design is an issue. For example, in two-dimensional (2D) fabrication, co-extrusion and reduction sintering [20] and microelectromechanical system fabrication [21] are used in experiments of negative thermal expansion materials. Additive manufacturing $(\mathrm{AM})$ or rapid prototyping techniques have been focused on recently $[22]$ to obtain detailed three-dimensional (3D) shapes. Such techniques have been used mainly in the development of scaffolds with matched physical properties with target body parts[23, 24, 25]. Among the AM techniques, selective laser melting (SLM), in which 3D structures are formed by melting and solidifying the material powder according to a 3D design using laser, is said to be able to achieve detailed and dense fabrication [26]. This could be a suitable fabrication method for porous or composite materials with optimized internal geometries. Several experimental verifications of the optimized internal geometries have been reported recently in elastic performances [27, 28, 29, 16, 30, 31]. Electron beam melting could be used in porous metal fabrication [32]. However, SLM has an advantage over electron beam melting in terms of the fineness of fabrication. As a special feature of SLM, metals could be handled as forming materials; plastics are the major forming materials in other AM methods. In addition to its use in structural 
parts because of the high metal stiffness and strength, there is an expectation for the use of optimized porous or composite materials in thermal conducting devices because of the larger metal thermal conductivity over plastics.

In this paper, we derive an optimal microstructural shape with high effective isotropic thermal conductivity. The effective physical properties of the internal geometries are calculated by using the averaging theorem [33, 19]. The internal geometry is designed to maximize the effective thermal conductivity by using numerical topology optimization. The obtained effective performance is compared with the HS bound of the isotropic thermal conductivity. The optimized structure is then fabricated using metal SLM, and the effective thermal conductivities of the test pieces are measured.

\section{Effective thermal conductivity and its theoretical bound}

\subsection{Equation of state of thermal conduction}

The static thermal conduction problem of porous material made from a metal with isotropic thermal conductivity is considered. The equation of state of the static thermal conduction of the body $\Omega$ with inhomogeneous Dirichlet boundary condition on the boundary $\Gamma$ is given as:

$$
\left\{\begin{array}{r}
-\nabla \cdot(\lambda \nabla T)=0 \text { in } \Omega \\
T=T_{0} \text { on } \Gamma
\end{array}\right.
$$

where $T$ is the temperature, $\lambda$ is the thermal conductivity, and $T_{0}$ is the boundary temperature $\Gamma$. By solving this equation numerically using finite element methods (FEM), the distribution of $T$ in $\Omega$ is obtained. 


\subsection{Calculation of effective thermal conductivity}

R1C1:In this research, porous materials composed of periodic structures are designed. The base pore structure is a cube as shown in Fig. 1, and is also used as the representative volume element (RVE) for calculating the effective thermal conductivity of the designed porous material. The effective thermal conductivity of the designed porous material is calculated based on the averaging theorem [33, 19]. In this method, the porous body is assumed to be statistically homogeneous (SH). A RVE with volume $\Omega$ is introduced to the body to calculate the effective physical property, assuming that the states of total body average and one of the RVE averages are the same in a SH physical field. The SH field in the RVE is generated by introducing boundary conditions that produce a homogenous field if the physical properties of the body are homogenous.

The effective thermal conductivity tensor $\boldsymbol{\lambda}^{*}$ is obtained by assuming that the homogenized energy and the RVE energy calculated directly from (1) are equal:

$$
\frac{1}{2} \boldsymbol{\lambda}^{*} \overline{\nabla T} \cdot \overline{\nabla T} V=\frac{1}{2} \int \lambda \nabla T \cdot \nabla T d x
$$

where $\overline{\nabla T}$ is the SH field of the temperature gradient. From (2), $\lambda_{11}^{*}$ is obtained by applying boundary conditions to produce $\overline{\nabla T}_{1} \neq 0$ and $\overline{\nabla T}_{2}=$ $\overline{\nabla T}_{3}=0 . \lambda_{22}^{*}$ and $\lambda_{33}^{*}$ are calculated in the same way. Other $\lambda_{i j}^{*}$ are calculated by introducing boundary conditions to produce only one $\overline{\nabla T}_{i}$ of zero. R1C1:We introduced a pair of Dirichlet boundary conditions with unit temperature gradient in the $i$ direction to have $\overline{\nabla T}_{i}=1$. Peri- 
odic boundary conditions were introduced to obtain $\overline{\nabla T}_{i}=0$. An example set of boundary conditions for calculating $\lambda_{11}^{*}$ is shown in Fig. 1.

Periodic porous material

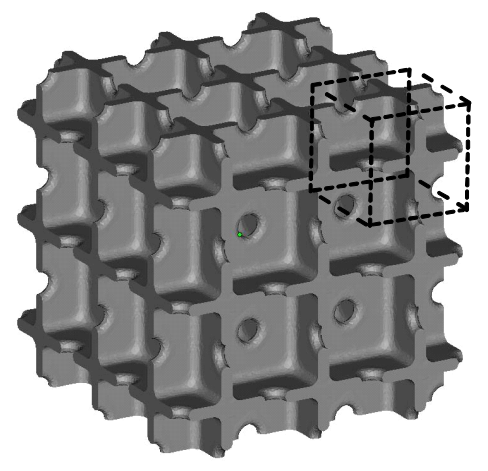

Representative Volume element

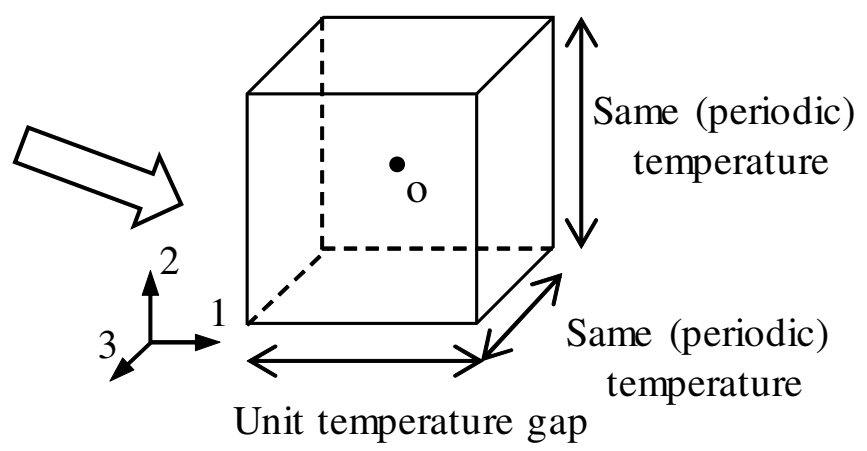

Figure 1: R1C1 and R1C4:Illustrations of a periodic porous material composed of unit length RVE. A pair of Dirichlet boundary conditions with unit temperature gradient is set in direction 1. Periodic boundary conditions are set in directions 2 and 3 , respectively.

The asymptotic homogenization method is also a major approach of effective material property calculation in the optimization of material internal geometry [34]. Although we introduced an averaging theorem-based method for its ease of implementation in finite element analysis code, our calculation results were consistent with those of the homogenization method.

\subsection{Hashin-Shtrikman bound}

As a criterion of extreme isotropic effective porous metal thermal conductivity, HS bounds, which are major theoretical bounds based on the variational principle, are introduced $[17,19]$. When the 3D two-phase composite 
material is composed of two original isotropic heat conductive materials with thermal conductivities $\lambda_{1}$ and $\lambda_{2}\left(\lambda_{1}>\lambda_{2}\right)$ and isotropic effective conductivity $\lambda^{*}$, the lower and upper bound of the effective thermal conductivity, $\lambda_{H S}^{-}$ and $\lambda_{H S}^{+}$, with respect to their volume fractions $f$ and $1-f$ is as follows:

$$
\begin{gathered}
\lambda_{H S}^{-}=\lambda_{2}-\frac{3 f \lambda_{2}\left(\lambda_{1}-\lambda_{2}\right)}{3 \lambda_{2}+(1-f)\left(\lambda_{1}-\lambda_{2}\right)} \\
\lambda_{H S}^{+}=\lambda_{1}-\frac{3(1-f) \lambda_{1}\left(\lambda_{1}-\lambda_{2}\right)}{3 \lambda_{1}-f\left(\lambda_{1}-\lambda_{2}\right)}
\end{gathered}
$$

By substituting the thermal conductivity of the original metal and air into $\lambda_{1}$ and $\lambda_{2}$, the extreme isotropic effective thermal conductivity of the porous metal with volume fraction $f$ is obtained from (4).

\section{Optimization methodology}

\subsection{Phase field-based topology optimization}

Phase-field-based topology optimization methods [35] are used to optimize the shape of the pore structure of the RVE because this method can perform fundamental optimizations over arbitrary domains including shape and topology; viz., the number of holes. R1C2: Different from the ordinary topology optimization of the material layout of the whole target domain, the phase field-based topology optimization changes the target shape according to the boundary movement. This is like shape optimizations, although its allows topology changes. There are two reasons why we have chosen the boundary variation method and not the usual whole domain variation method. Sometimes, problems are encountered related to gray unclear domains in topology optimization. It is difficult to identify whether these domains belong to an optimal shape or to a void. 
Second, complicated shapes, which can be generated by usual topology optimization, are not suitable for fabrication by additive manufacturing. In the phase field method, because of the inherent perimeter minimization effect, complicated surface shapes can be avoided.

In the phase field method, the RVE shape is represented as a distribution of the density function. Optimization is performed by representing the physical property as a function of the density function $\phi$. R1C3: The local micro-scale thermal conductivity $\lambda$ in the porous material composed of a metal with thermal conductivity $\lambda_{1}$ and air with a thermal conductivity $\lambda_{2}$ is expressed in terms of the density function $\phi, \quad(0 \leq \phi \leq 1)$ :

$$
\lambda=\lambda_{2}+\phi^{p}\left(\lambda_{1}-\lambda_{2}\right) .
$$

The optimum shape of the pore structure can then be specified as a distribution of $\phi$. The density function $\phi$ is updated by solving the following partial differential Allen-Cahn equation:

$$
\frac{\partial \phi}{\partial t}=\kappa \nabla^{2} \phi-P^{\prime}(\phi)
$$

where

$$
\begin{gathered}
P(\phi)=\frac{1}{4} Q(\phi)+\eta R(\phi), \\
Q(\phi)=\phi^{2}\left(1-\phi^{2}\right), \\
R(\phi)=\phi^{3}\left(6 \phi^{2}-15 \phi+10\right),
\end{gathered}
$$

and where $t$ is the artificial time that corresponds to a step size of the design variable, $P$ is the asymmetric double-well potential, and $\eta$ is a positive coefficient. $P$ has two minima, at 0 and 1 . Because of coupling between the 
diffusion and reaction terms in (6), the density function $\phi$ is divided into several domains that correspond to the value 0 or 1 . The so-called phase field interface that corresponds to the intermediate values $0<\phi<1$ exists between these domains. The interface moves in the normal direction according to the shape of the double-well potential. That is, the interface evolves in the direction of lower minimum potential.

To obtain the optimal RVE shape with an isotropic heat conductive performance close to the HS bound, we aim to maximize the effective thermal conductivities in three directions. R1C4: We achieve isotropy by setting the mirror symmetric structure over the nine planes shown in Fig. 2. Thus, the distribution of the density function is optimized only on the tetrahedral domain with a volume 1/64 that of the base domain shown in Fig. 2. The entire shape is constructed by copying this domain. The optimization problem is then formulated with an added RVE volume fraction constraint as follows:

$$
\operatorname{minimize}-\lambda_{i i}^{*}(i=1,2,3)
$$

subject to

$$
\begin{aligned}
& \text { Eqs. (1) and (2), } \\
& \begin{array}{l}
\frac{\int_{\Omega} \phi \mathrm{d} x}{\int_{\Omega} \mathrm{d} x} \leq v f_{\max }, \\
0 \leq \phi \leq 1,
\end{array}
\end{aligned}
$$

where $v f_{\max }$ is the maximum volume fraction of the RVE. 


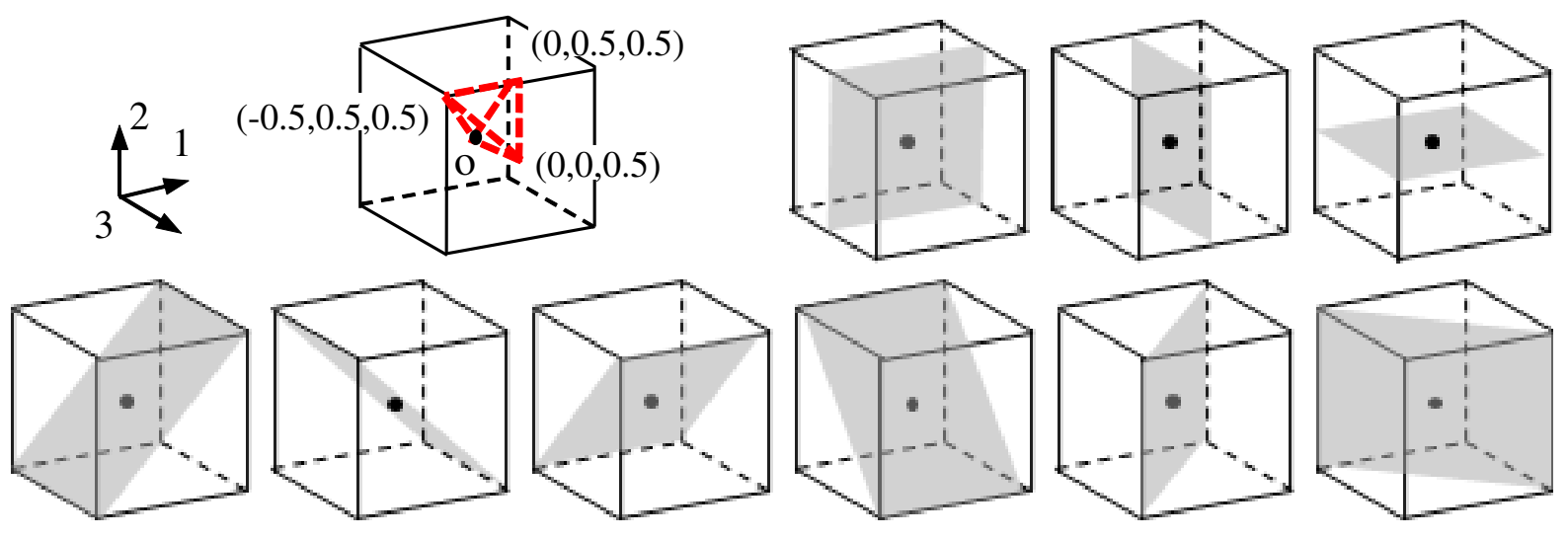

Figure 2: R1C1 and R1C4:Illustrations of the design domain in the RVE. The red dotted lines of the upper left RVE indicate the optimized design of the unit length RVE when considering symmetry. This is a tetrahedral composed of four vertices, RVE center, $(0,0,0.5),(0,0.5,0.5)$ and $(-0.5,0.5,0.5)$ when the original point is located on the center. The other nine RVEs represent the planes of symmetry. The first three planes are 1-2, 2-3 and 1-3 planes. The other six planes are diagonal planes perpendicular to these.

\subsection{Numerical implementation and optimization algorithm}

The density function is updated using gradient-based algorithms, and the sensitivities for the objective function and the constraint must be calculated. R1C5: The sensitivity of the effective thermal conductivity used in the objective function in (10) can be expressed in terms of the functions of the state variable as:

$$
\lambda_{11,22 \text { or } 33}^{* \prime}(\phi)=-\lambda^{\prime}(\phi) \nabla T \nabla T
$$

The detailed derivation is given in the Appendix.

Figure 3 shows a flowchart of the optimization procedure. The temperature distribution in the RVE is calculated by solving (1) by FEM. Then, the 
objective function (effective thermal conductivity) and the constraint (volume fraction of RVE) are calculated. Sensitivities for the objective function and the constraint are also calculated. Finally, the density functions are updated by solving (6) using the finite difference method. These procedures are repeated until the iteration limit is reached or until certain specified convergence criteria hold.

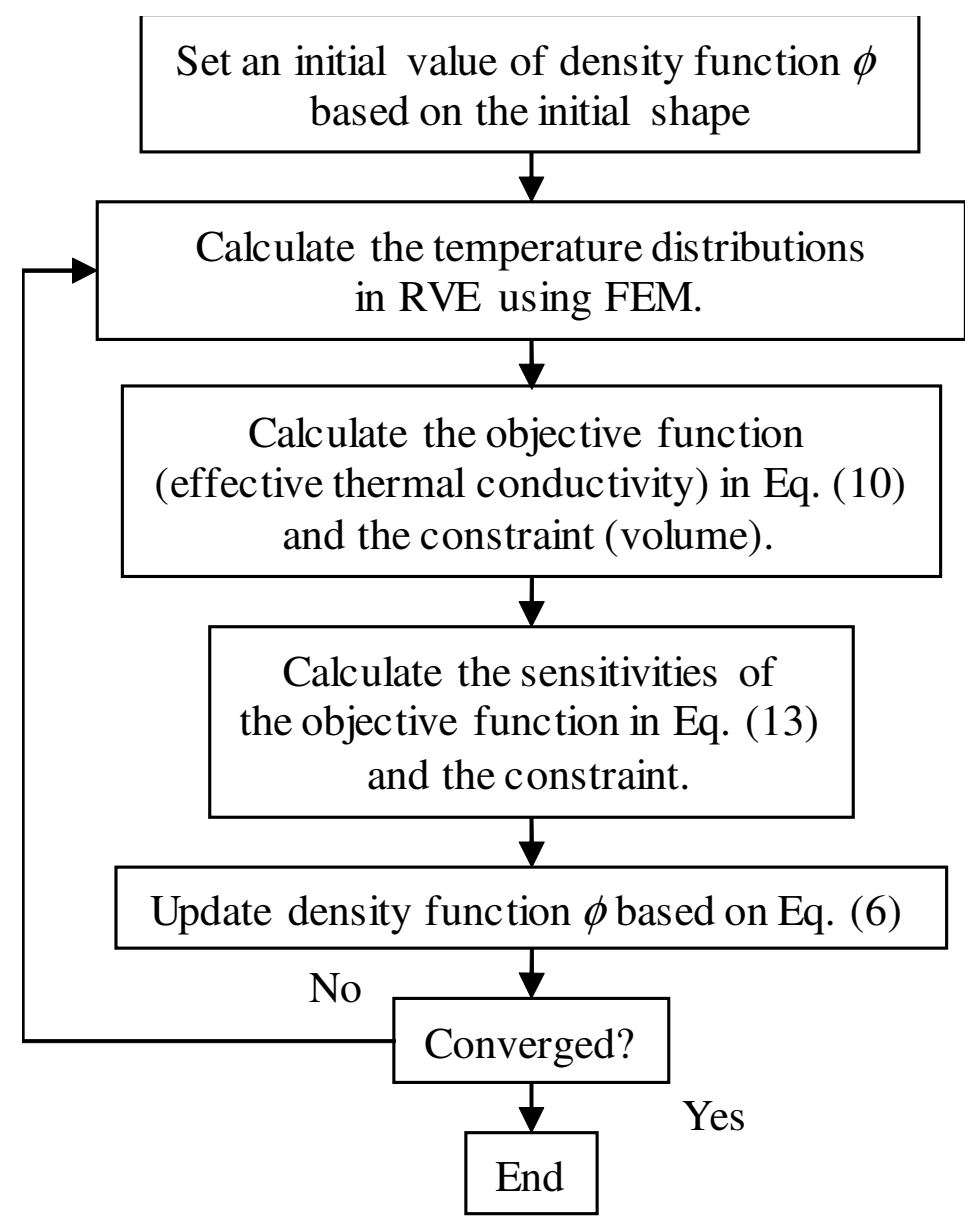

Figure 3: Flowchart of the optimization procedure. 


\section{Selective laser melting}

To fabricate the 3D optimized pore structure, the SLM process is introduced. An outline of the process is shown in Fig. 4. Input 3D data are divided into sliced data that represent $2 \mathrm{D}$ shapes on each layer. The $3 \mathrm{D}$ shape is achieved by stacking the $2 \mathrm{D}$ layers made from sintered metal. Building of the 2D layer is achieved by spreading the metal powder and by laser sintering according to the sliced data.

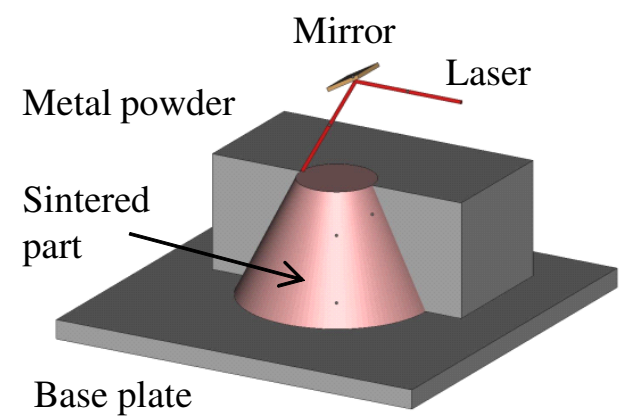

Figure 4: Outline of selective laser sintering process.

Although this process provides flexible manufacturability even for relatively complicated shapes for which fabrication is impossible using conventional metal fabrication techniques such as casting and forging, a few restrictions exist for the fabricated design. First, the design must have a hole to remove the remaining metal powder; when a porous structure is constructed, it must be an open cell structure. Second, the inclination angle from the vertical is restricted as the intended layer shape may be distorted because of a lack of stiffness when the new layer protrudes significantly from the side of the previous layer. The supporting structure that must be removed after finishing the SLM process is usually added to fabricate such a structure [36]. 
However, in the fabrication of small structures such as pore structures, removal of the added supporting structure is rather difficult. Thus, too many inclination shapes are unacceptable for fabrication even if they achieve good numerical performance.

\section{Numerical study}

Some numerical studies were performed to generate optimal 3D shapes of pore structure and achieve high isotropic effective thermal conductivities with specified volume fractions. A virtual material with unit thermal conductivity $\lambda=1$ was introduced for optimization. The RVE was set as a unit cube. Non-design domain (or fixed) holes (0.2 diameter) were introduced at the center of each plane of the RVE as powder-removing holes. Other domains in the RVE were handled as a design domain. The pair of Dirichlet boundary conditions $T=0$ on $\Gamma_{i 1}$ and $T=1$ on $\Gamma_{i 2}$ were introduced as the homogeneous boundary condition-generating SH field $\overline{\nabla T}_{i}=1$ used in the calculation of the effective thermal conductivity in (2). $\Gamma_{i 1}$ and $\Gamma_{i 2}$ are the two facing boundaries of the RVE in the $i$-direction. To generate $\overline{\nabla T}_{i}=0$, a pair of periodic boundary conditions was introduced on $\Gamma_{i 1}$ and $\Gamma_{i 2}$. R1C7: These boundary conditions for calculating $\lambda_{11}^{*}$ are illustrated in Fig. 5 as an example. R1C9: The RVE domain was meshed using a $32 \times 32 \times 32$ cubic finite-element mesh for the calculation of the temperature field. First-order elements were used for the formulation of each element. FEM was performed using commercial software Comsol Multiphysics for effective computation using a multicore processor [37]. R1C8: The density function was discretized using the second- 
order finite element on the design domain. The reference points of the density function were set on each node of the mesh, including internal ones. The temperature field and density function meshes were connected, based on the concept of multi-resolution topology optimization [38].

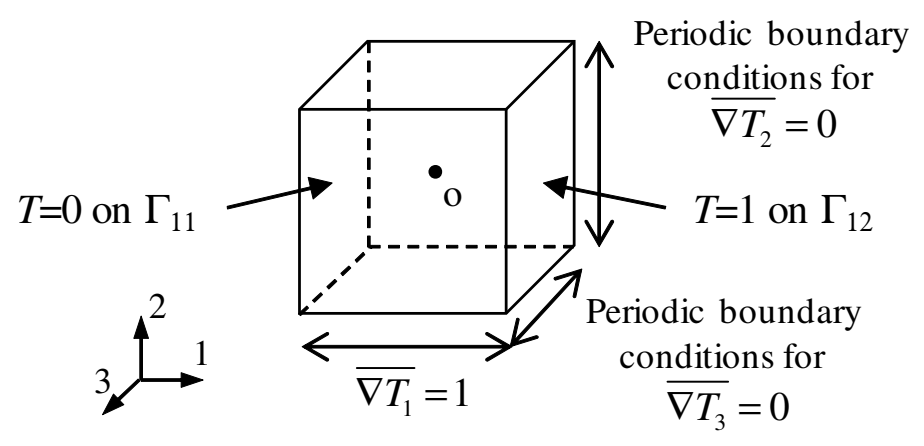

Figure 5: Boundary conditions for calculating $\lambda_{11}^{*}$.

According to existing research on the optimization of effective isotropic conductivity, the optimal shapes of the RVE could be simple, closed-cell structures of any volume fraction $[6,14]$. Thus, as a general initial shape for the optimization, a closed-cell structure with 0.9 diameter spherical air hole was introduced. It forms an open-cell structure because of the powderremoving holes. R1C10: Fig. 6 shows the initial shapes and hole locations. For clarity, the displaying structure is selected from the repeated RVE at point $(1,1,1)$ of the original RVE.

Optimizations are performed to maximize the objective function in (10) for $0.3,0.4,0.5,0.6$, and 0.7 volume fractions. To study performance reduction by the powder-removing holes, optimizations were also performed without fixed holes. Figures 7 and 8 show the 0.5 isosurface of the optimal 


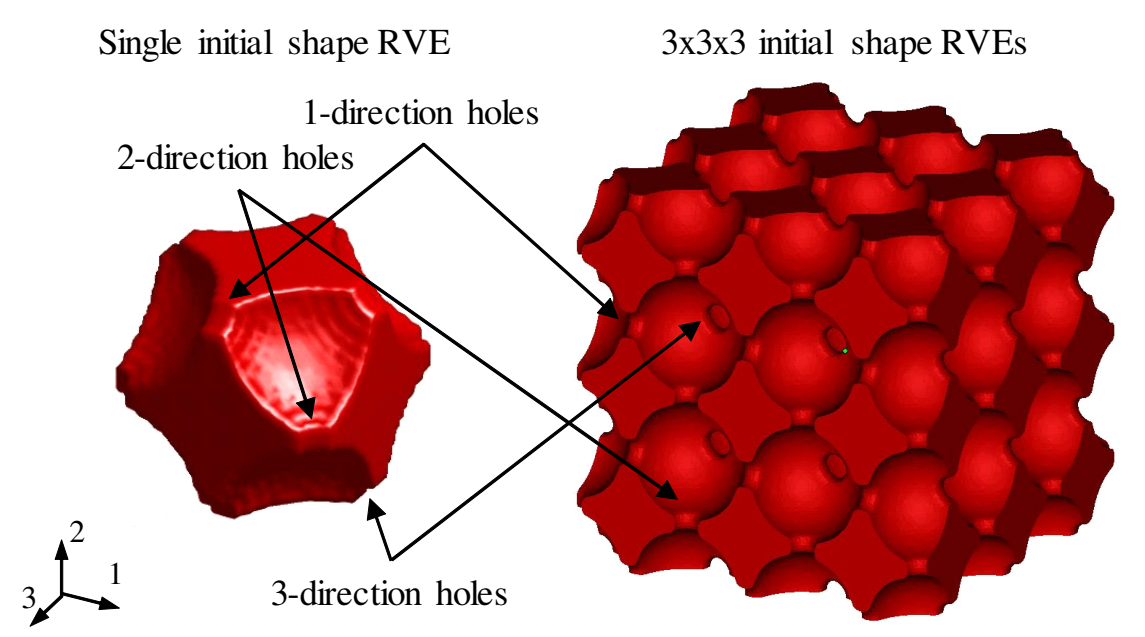

Figure 6: R1C10: Initial RVE shapes and the location of the powder-removing holes.

density distribution in each volume fraction after 200 optimization iterations with and without a hole non-design domain, respectively. Similar to existing research $[6,14]$, in low volume fractions, optimal shapes have cube-like air holes. Corners were smoothed for isotropic high conductivity. In high volume fractions, however, the optimal shapes have sphere-like air holes. The existence of powder-removing holes did not affect the optimal shape significantly. Table 1 summarizes the performance of the optimal configurations. Approximately $99 \%$ and $96 \%$ of the HS upper bound performance was obtained with and without the powder-removing holes, respectively. Optimal results close to the theoretical limit appear to have been obtained. 


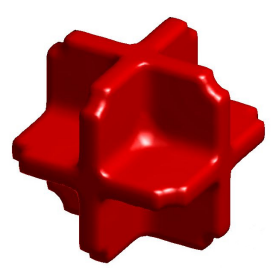

(a)

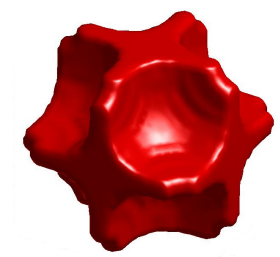

(b)

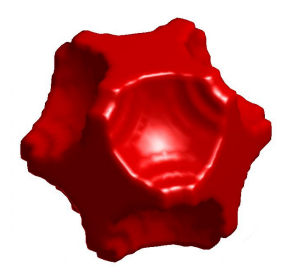

(c)

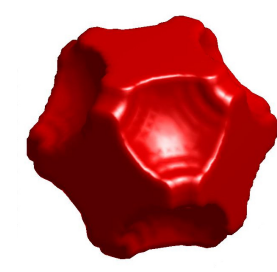

(d)

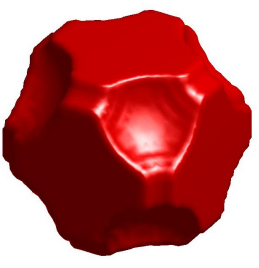

(e)

Figure 7: Optimal configuration of RVE with powder-removing holes. (a) 30\%, (b) 40\%, (c) $50 \%$, (d) $60 \%$, and (e) $70 \%$ host material volume fractions.

\section{Experimental study}

\subsection{Measurement of effective thermal conductivity}

An experimental study was conducted to form porous metal with extreme effective isotropic thermal conductivity. R1C12:The effective thermal conductivity was measured using a steady-state method and calculated from the one-dimensional (1D) temperature gradient and the heat flux of the test piece [39]. It regarded the temperature distribution and the thermal conductivity as homogeneous inside the test piece, although it would not be accurate. An outline of the experimental equipment is shown in Fig. 9. The test piece was sandwiched 


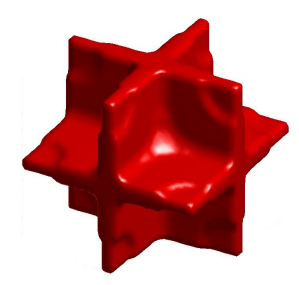

(a)

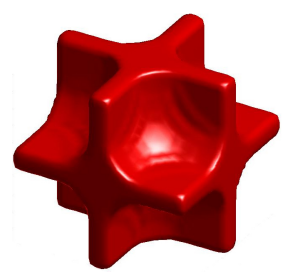

(b)

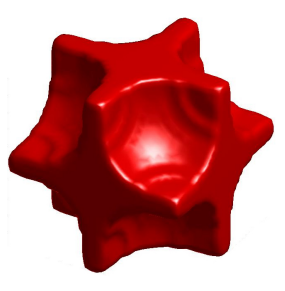

(c)

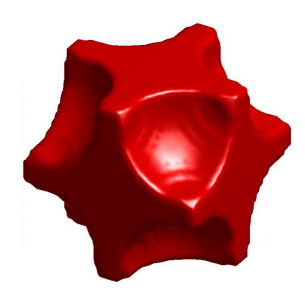

(d)

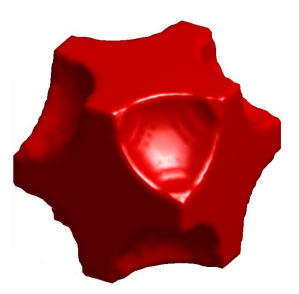

(e)

Figure 8: Optimal configuration of RVE without powder-removing holes. (a) 30\%, (b) $40 \%$, (c) $50 \%$, (d) $60 \%$, and (e) $70 \%$ host material volume fractions.

between reference brass bars. At steady state, the 1D temperature distribution was measured using thermocouples at several points on the brass bars. The $1 \mathrm{D}$ temperature gradient $\Delta T$ of the test piece and the heat flux $q$ applied to the test piece was calculated from the position and temperature of the thermocouples. The effective thermal conductivity of the test piece $\lambda_{\text {Test }}$ was calculated using these values and the height $\Delta x$ of the test piece:

$$
\lambda_{\text {Test }}=\frac{q}{\Delta T} \Delta x
$$


Table 1: Summary of effective thermal conductivities of optimization results.

\begin{tabular}{c|ccccc} 
Volume fraction & HS upper bound & $\begin{array}{c}\text { Optimal porous } \\
\text { with holes }\end{array}$ & $\begin{array}{c}\text { Ratio to } \\
\text { HSper bound }\end{array}$ & $\begin{array}{c}\text { Optimal porous } \\
\text { without holes }\end{array}$ & $\begin{array}{c}\text { Ratio to } \\
\text { HSper bound }\end{array}$ \\
\hline \hline $30 \%$ & 0.224 & 0.216 & $96.6 \%$ & 0.221 & $98.9 \%$ \\
$40 \%$ & 0.309 & 0.294 & $95.1 \%$ & 0.307 & $99.4 \%$ \\
$50 \%$ & 0.401 & 0.384 & $95.7 \%$ & 0.399 & $99.5 \%$ \\
$60 \%$ & 0.501 & 0.481 & $96.0 \%$ & 0.500 & $99.8 \%$ \\
$70 \%$ & 0.610 & 0.588 & $96.4 \%$ & 0.611 & $100.2 \%$
\end{tabular}

where

$$
q=\lambda_{\text {Brass }} \frac{\partial T}{\partial x}
$$

and $\lambda_{\text {Brass }}$ is the thermal conductivity of the reference brass bar. In this measurement, the heat flux $q$ of the test piece is calculated as the averaged heat flux of the upper and lower reference bars. If we assume that the reference bars and test piece are completely thermally insulated, the heat fluxes could be the same. R1C11: The basic temperature of the high- and low-temperature sides are $65^{\circ} \mathrm{C}$ and $12^{\circ} \mathrm{C}$. Radiative conduction can be ignored in such a low temperature range [40].

\subsection{Fabrication by selective laser melting}

The test pieces were fabricated based on the results for a representative $0.3,0.5$, and 0.7 volume fraction. Stereo lithography files of the RVEs were created based on the 0.5 isosurfaces of the optimal density distribution in Fig. 7. The size of each RVE was set to $5 \mathrm{~mm}$ with a periodic layout periodically in 3D . Cylindrical test pieces $(\phi 50 \mathrm{~mm} \times 20 \mathrm{~mm})$ were then clipped from the periodic structure. R1C11: In this pore size $(<\mathbf{1 0 m m})$, the convection effect can be ignored [40]. 


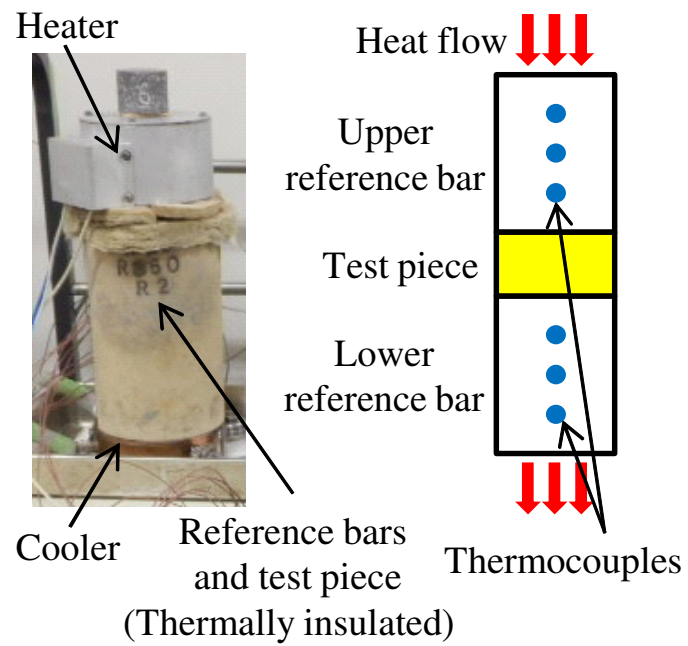

Figure 9: Photograph of measurement device and schematic of the thermally insulated section. The reference bars are brass and their thermal conductivities are 110 and 117 $\mathrm{W} /(\mathrm{m} \cdot \mathrm{K})$. The brass bar and test piece diameter is $5 \mathrm{~mm}$. The height of the brass reference bars and test piece are $60 \mathrm{~mm}$ and $20 \mathrm{~mm}$, respectively. Six thermocouples were placed in the cross-sectional centers of each brass bar at $10 \mathrm{~mm}$ intervals. The basic temperature of the high- and low-temperature sides are $65^{\circ} \mathrm{C}$ and $12^{\circ} \mathrm{C}$.

The models were fabricated using a SLM manufacturing device, EOS, EOSINT M 280 with 400 W fiber laser. EOS Maraging Steel MS1 was used, which is an Fe-based alloy that contains approximately $18 \% \mathrm{Ni}, 9 \%$ alloy and $6.7 \%$ other materials. The expected thermal conductivity without age hardening is approximately $15 \pm 0.8 \mathrm{~W} /(\mathrm{m} \cdot \mathrm{K})$. Figure 10 shows the fabricated test piece. The 3D models were authentic reproductions. The test piece masses were $95.6 \mathrm{~g}, 154.8 \mathrm{~g}$, and $218.8 \mathrm{~g}$. The density of the material measured from a bulk test piece was $8.0 \mathrm{~g} / \mathrm{cm}^{3}$. The relative densities of the $0.3,0.5$, and 0.7 volume fraction test pieces were $30.5 \%, 49.4 \%$, and $69.8 \%$ of the bulk material. The design volume differed slightly from the expected value because of 
the variation in density of the original material, which is unique to the SLM.

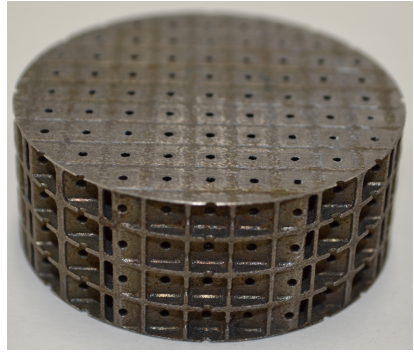

(a)

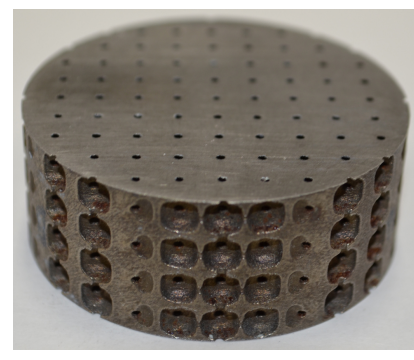

(b)

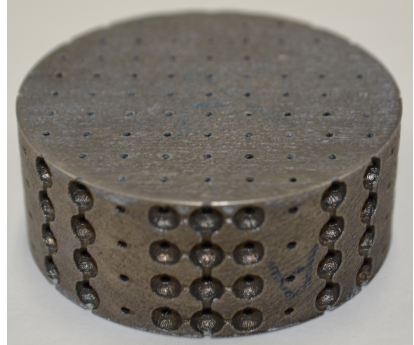

(c)

Figure 10: Photographs of test pieces with (a) 30\%, (b) 50\%, and (c) $70 \%$ volume fractions.

The internal shape of the structure was checked using $225 \mathrm{kV}$ X-rays as performed in [27]. Only the 0.3 volume fraction test piece was tested because of the power limit. Cross sections were obtained from the bottom to the top of the cylinder at $0.1 \mathrm{~mm}$ intervals. Representative pictures taken at a half and quarter of the RVE are shown in Fig. 11. Clear shapes without unexpected cavities or solids were obtained. Such disorders were not visible in any other cross sections.

\subsection{Experimental results}

The effective conductivities of the test pieces were measured. To obtain the thermal conductivity of the original material, the bulk test piece was also prepared using the same SLM. Table 2 summarizes the experimental results obtained from the measured temperatures shown in Fig. 12. The thermal conductivity of the bulk test piece is $14.39 \mathrm{~W} /(\mathrm{m} \cdot \mathrm{K})$. R1C13: Using this value for the host material, the errors between the simulated and 
(b)

(c)

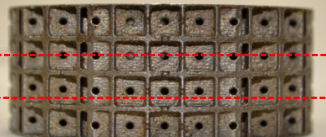

(a)
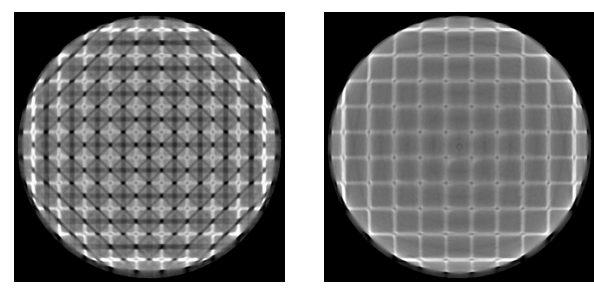

(b)

(c)

Figure 11: X-ray images of $30 \%$ volume fraction test piece produced using a MMT-225 machine, Shimadzu Corporation. (a) Position of cross sections (b) and (c).

measured effective thermal conductivities are $3.23 \%$ in the 0.3 volume fraction test piece, $-4.47 \%$ in the 0.5 volume fraction test piece and $-\mathbf{4 . 0 9 \%}$ in the 0.7 volume fraction test piece. These values agree relatively well, whereas the measurement of effective elastic properties of the additive manufactured topology of the optimized material showed an approximate $46 \%$ [27] and $76 \%$ error [28] between the simulated and experimental results in related research. The manufacturing accuracy could have caused this improvement. In previous research, clear differences were observed between the intended shape and fabricated test piece, such as the existence of a deposit and a reduction in surface curvature. As shown in Figs 10 and 11, shape disorder thorough fabrication was small in this research because 
of the slightly larger RVE size that was used in this research and a recent performance development of the SLM device. In particular, we confirmed through X-ray testing the absence of unexpected voids that reduce thermal properties. Table 2 also shows the error between the measured results and the HS upper bound. These results are plotted on the HS upper bound curve in Figure 13. As a result, the effective isotropic thermal conductivity close to the HS upper bound within 10\% error could be achieved by metal SLM. To confirm the advantages of designed porosity over conventional random cell porosity, Bruggeman's approximation [41], which shows good agreement with random open cell aluminum forms with $0.5-0.8$ porosity [42], is also plotted. A $35.8 \%$ advantage existed in the 0.3 volume fraction.

$$
f=\left(\frac{\lambda_{2}-\lambda^{*}}{\lambda_{2}-\lambda_{1}}\right)\left(\frac{\lambda_{1}}{\lambda^{*}}\right)^{1 / 3}
$$




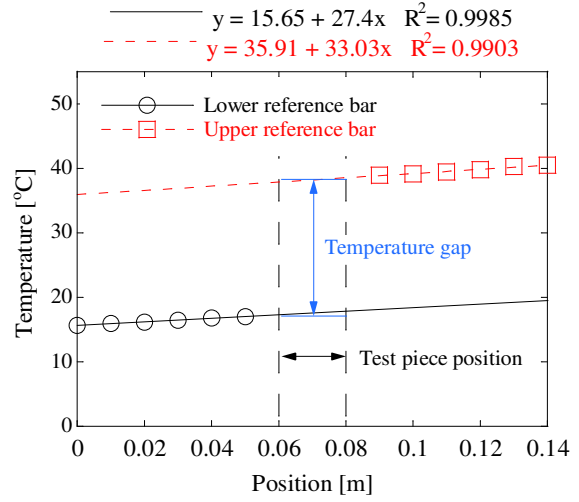

(a)

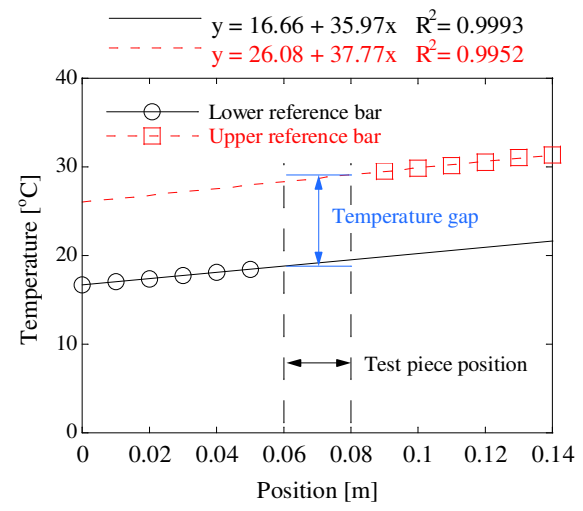

(c)

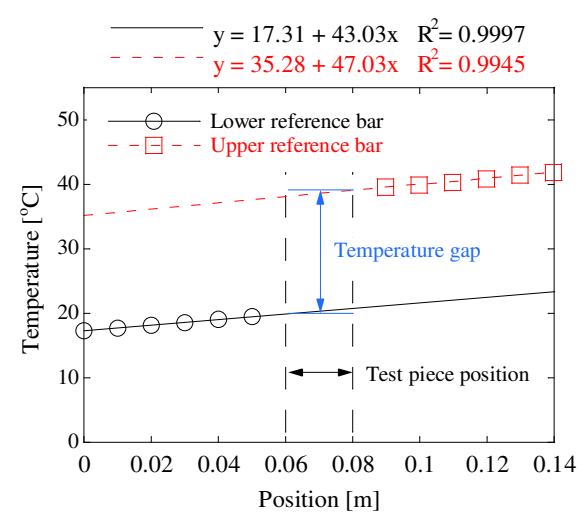

(b)

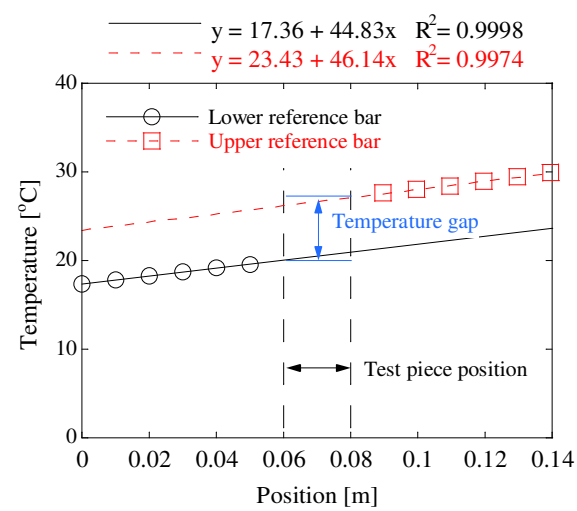

(d)

Figure 12: Temperature measurement of volume fraction (a) $30 \%$, (b) $50 \%$, (c) $70 \%$, and (d) bulk test pieces. 
Table 2: Summary of measured and simulated effective thermal conductivities.

\begin{tabular}{c|lc|l|l|} 
Volume fraction & $\begin{array}{l}\text { Measured } \\
{[\mathrm{W} /(\mathrm{m} \cdot \mathrm{K})]}\end{array}$ & value & $\begin{array}{l}\text { Error over the simu- } \\
\text { lated value [\%] }\end{array}$ & $\begin{array}{l}\text { Error over the HS } \\
\text { bound [\%] }\end{array}$ \\
\hline \hline $30 \%$ & 3.21 & 3.23 & -0.31 \\
$50 \%$ & 5.28 & -4.47 & -8.55 \\
$70 \%$ & 8.11 & -4.09 & -7.54 \\
Bulk & 14.39 & 0.00 & 0.00
\end{tabular}

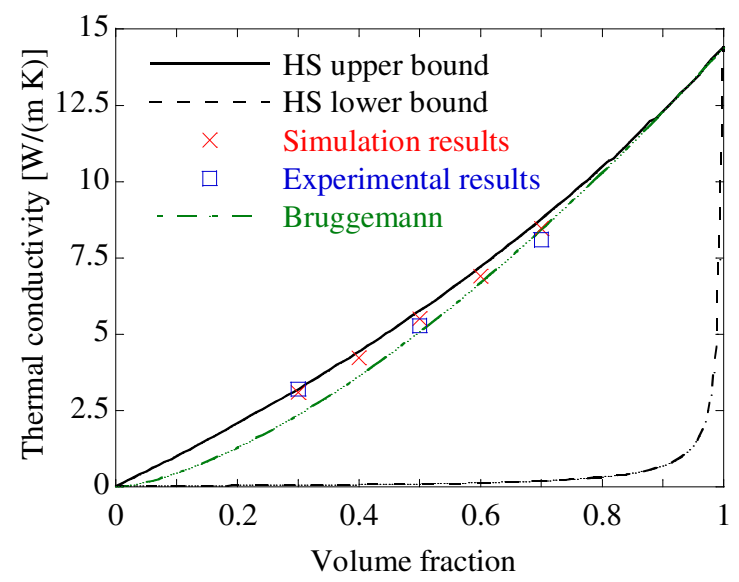

Figure 13: Simulated and experimental results of effective thermal conductivities of optimal pore structure plotted with HS bounds and Bruggeman's approximation. HS bounds, simulated results, and Bruggeman's approximation were calculated using the measured thermal conductivity of the bulk test piece $14.39 \mathrm{~W} /(\mathrm{m} \cdot \mathrm{K})$ and the thermal conductivity of air $0.0257 \mathrm{~W} /(\mathrm{m} \cdot \mathrm{K})$.

\section{Conclusion}

We studied the validity of the effective thermal conductivity of porous metal with optimized pore structure. The effective thermal conductivity was calculated based on statically homogenized theory. The pore structure was optimized based on topology optimization by maximizing the effective 
thermal conductivity as the objective function. The performance of the optimal pore structures was approximately $95 \%$ of the HS upper bound of the isotropic thermal conductivity. The optimal structures were fabricated as porous maraging steel using metal SLM. The shape of the fabricated test piece was checked by X-ray analysis. The effective thermal conductivities of the fabricated porous material were measured by a steady-state method. Errors between the experimental and numerical results were $-4.47 \%$ to $3.23 \%$. Errors between the experimental results and HS upper bounds were $-8.55 \%$ to $-0.31 \%$. By comparison with Bruggeman's approximation, the proposed designed porous material appears to have an advantage over conventional porous materials in terms of their relatively high porosity. However, in the fabrication of high porosity materials by SLM, fabrication restrictions on minimum part size in manufacturing must be considered.

Some limitations exist in the shape handled by SLM in addition to requirements of powder-removing holes and minimum and maximum part sizes. These include limitations of manufacturable inclination angle and bridging length [36]. Although the optimal design generated in this research did not conflict with these limitations, the design to minimize performance reduction because of these limitations must be an important issue in porous or material design through microstructural shape. R1C11: Moreover, the effect of convection and radiation could be ignored under the smallpore-size and low-temperature experimental conditions of this research. However, the optimization of large-pore-size materials for high-temperature environments considering both effects would be interesting future work. 
We provide the STL file of the developed pore structure as supplementary material and expect further development, experimentation and utilization in industrial applications. Contact the corresponding author in this regard.

\section{Acknowledgments}

This work was supported by JSPS KAKENHI Grant number 15K12557.

\section{References}

[1] S. B. Vigdergauz, Regular structures with extremal elastic properties, Mech. Solid. 24 (3) (1989) 57-63.

[2] S. B. Vigdergauz, Three-dimensional grained composites of extreme thermal properties, J. Mech. Phys. Solid. 42 (5) (1994) 729-740.

[3] O. Sigmund, A new class of extremal composites, J. Mech. Phys. Solid. 48 (2) (2000) 397-428.

[4] S. Torquato, S. Hyun, A. Donev, Multifunctional composites: optimizing microstructures for simultaneous transport of heat and electricity, Phys. Rev. Lett. 89 (26) (2002) 266601.

[5] J. K. Guest, J. H. Prévost, Optimizing multifunctional materials: design of microstructures for maximized stiffness and fluid permeability, Int. J. Solid. Struct. 43 (22) (2006) 7028-7047.

[6] V. J. Challis, A. P. Roberts, A. H. Wilkins, Design of three dimensional isotropic microstructures for maximized stiffness and conductivity, Int. J. Solid. Struct. 45 (14) (2008) 4130-4146. 
[7] R. Lakes, Foam structures with a negative poisson's ratio, Science 235 (4792) (1987) 1038-1040.

[8] U. D. Larsen, O. Sigmund, S. Bouwstra, Design and fabrication of compliant micromechanisms and structures with negative poisson's ratio, J. Microelectromech. Syst. 6 (2) (1997) 99-106.

[9] R. Lakes, Cellular solid structures with unbounded thermal expansion, J. Mater. Sci. Lett. 15 (6) (1996) 475-477.

[10] O. Sigmund, S. Torquato, Composites with extremal thermal expansion coefficients, Appl. Phys. Lett. 69 (21) (1996) 3203-3205.

[11] M. P. Bendsøe, N. Kikuchi, Generating optimal topologies in structural design using a homogenization method, Comput. Meth. Appl. Mech. Eng. 71 (2) (1988) 197-224.

[12] M. P. Bendsøe, O. Sigmund, Topology Optimization: Theory, Methods, and Applications, Springer-Verlag, Berlin, 2003.

[13] S. Torquato, Random heterogeneous materials: microstructure and macroscopic properties, Springer, 2002.

[14] S. Zhou, J. Cadman, Y. Chen, W. Li, Y. M. Xie, X. Huang, R. Appleyard, G. Sun, Q. Li, Design and fabrication of biphasic cellular materials with transport properties-a modified bidirectional evolutionary structural optimization procedure and matlab program, Int. J. Heat Mass. Tran. 55 (25) (2012) 8149-8162. 
[15] J. E. Cadman, S. Zhou, Y. Chen, Q. Li, On design of multi-functional microstructural materials, J. Mater. Sci. 48 (1) (2013) 51-66.

[16] Y. M. Xie, X. Yang, J. Shen, X. Yan, A. Ghaedizadeh, J. Rong, X. Huang, S. Zhou, Designing orthotropic materials for negative or zero compressibility, Int. J. Solid. Struct. 51 (23) (2014) 4038-4051.

[17] Z. Hashin, S. Shtrikman, A variational approach to the theory of the effective magnetic permeability of multiphase materials, J. Appl. Phys. 33 (10) (1962) 3125-3131.

[18] Z. Hashin, S. Shtrikman, A variational approach to the theory of the elastic behavior of multiphase materials, J. Mech. Phys. Solids 11 (2) (1963) 127-140.

[19] Z. Hashin, Analysis of composite materials - a survey, J. Appl. Mech. 50 (3) (1983) 481-505.

[20] J. Qi, J. W. Halloran, Negative thermal expansion artificial material from iron-nickel alloys by oxide co-extrusion with reductive sintering, J. Mater. Sci. 39 (13) (2004) 4113-4118.

[21] N. Yamamoto, E. Gdoutos, R. Toda, V. White, H. Manohara, C. Daraio, Thin films with ultra-low thermal expansion, Adv. Mater. 26 (19) (2014) 3076-3080.

[22] I. Gibson, D. Rosen, B. Stucker, Additive manufacturing technologies, Springer, 2010. 
[23] S. J. Hollister, R. D. Maddox, J. M. Taboas, Optimal design and fabrication of scaffolds to mimic tissue properties and satisfy biological constraints, Biomaterials 23 (20) (2002) 4095-4103.

[24] S. J. Hollister, Porous scaffold design for tissue engineering, Nat. Mater. 4 (7) (2005) 518-524.

[25] M. Castilho, M. Dias, U. Gbureck, J. Groll, P. Fernandes, I. Pires, B. Gouveia, J. Rodrigues, E. Vorndran, Fabrication of computationally designed scaffolds by low temperature 3 d printing, Biofabrication 5 (3) (2013) 035012.

[26] W. E. Frazier, Metal additive manufacturing: A review, J. Mater. Eng. Perform. 23 (6) (2014) 1917-1928.

[27] C. Y. Lin, T. Wirtz, F. LaMarca, S. J. Hollister, Structural and mechanical evaluations of a topology optimized titanium interbody fusion cage fabricated by selective laser melting process, J. Biomed. Mater. Res. 83 (2) (2007) 272-279.

[28] D. Xiao, Y. Yang, X. Su, D. Wang, J. Sun, An integrated approach of topology optimized design and selective laser melting process for titanium implants materials, Bio-Med. Mater. Eng. 23 (5) (2013) 433-445.

[29] E. Andreassen, B. S. Lazarov, O. Sigmund, Design of manufacturable 3d extremal elastic microstructure, Mech. Mater. 69 (1) (2014) 1-10.

[30] A. Takezawa, M. Kobashi, M. Kitamura, Porous composite with negative thermal expansion obtained by photopolymer additive manufacturing, APL Mater. 3 (7) (2015) 076103. 
[31] P. Zhang, J. Toman, Y. Yu, E. Biyikli, M. Kirca, M. Chmielus, A. C. To, Efficient design-optimization of variable-density hexagonal cellular structure by additive manufacturing: Theory and validation, J. Manuf. Sci. Eng. 137 (2) (2015) 021004.

[32] J. Schwerdtfeger, F. Wein, G. Leugering, R. F. Singer, C. Körner, M. Stingl, F. Schury, Design of auxetic structures via mathematical optimization, Adv. Mater. 23 (22-23) (2011) 2650-2654.

[33] R. Hill, On constitutive macro-variables for heterogeneous solids at finite strain, Proc. Roy. Soc. Lond. Math. Phys. Sci. 326 (1972) 131-147.

[34] J. M. Guedes, N. Kikuchi, Preprocessing and postprocessing for materials based on the homogenization method with adaptive finite element methods, Comput. Meth. Appl. Mech. Eng. 83 (2) (1990) 143-198.

[35] A. Takezawa, S. Nishiwaki, M. Kitamura, Shape and topology optimization based on the phasefield method and sensitivity analysis, J. Comput. Phys. 229 (7) (2010) 2697-2718.

[36] M. Leary, L. Merli, F. Torti, M. Mazur, M. Brandt, Optimal topology for additive manufacture: A method for enabling additive manufacture of support-free optimal structures, Mater. Des. 63 (2014) 678-690.

[37] L. H. Olesen, F. Okkels, H. Bruus, A high-level programming-language implementation of topology optimization applied to steady-state navierstokes flow, Int. J. Numer. Meth. Eng. 65 (7) (2006) 975-1001.

[38] T. Nguyen, G. Paulino, J. Song, C. Le, A computational paradigm for 
multiresolution topology optimization (mtop), Struct. Multidisc. Optim. 41 (4) (2010) 525-539.

[39] T. M. Tritt, D. Weston, Measurement techniques and considerations for determining thermal conductivity of bulk materials, in: T. M. Tritt (Ed.), Thermal Conductivity: theory, properties, and applications, Springer, 2004, pp. 187-203.

[40] T. W. Clyne, I. O. Golosnoy, J. C. Tan, A. E. Markaki, Porous materials for thermal management under extreme conditions, Phil. Trans. Math. Phys. Eng. Sci. 364 (2006) 125-146.

[41] P. G. Collishaw, J. R. G. Evans, An assessment of expressions for the apparent thermal conductivity of cellular materials, J. Mater. Sci. 29 (9) (1994) 2261-2273.

[42] E. Solórzano, J. A. Reglero, M. A. Rodríguez-Pérez, D. Lehmhus, M. Wichmann, J. A. De Saja, An experimental study on the thermal conductivity of aluminium foams by using the transient plane source method, Int. J. Heat. Mass. Tran. 51 (25) (2008) 6259-6267.

[43] G. Allaire, Conception Optimale De Structures, Springer-Verlag, Berlin, 2007.

\section{Appendix A. Sensitivity analysis}

A derivation of the sensitivity of the objective function in Eq. (10) is outlined according to the procedure described in Chapter 5 of [43]. 
The weak form of the linear thermal conduction equation in Eq. (1) is obtained after introducing a new function $\tilde{T}=T-T_{0}$ that satisfies $\tilde{T}$ on the boundary $\Gamma$ as follows:

$$
a(\tilde{T}, \bar{T})=-l(\bar{T})
$$

where

$$
\begin{gathered}
a(\tilde{T}, \bar{T})=\int \lambda \nabla T \cdot \nabla \bar{T} d x \\
l(\bar{T})=\int \lambda \nabla T_{0} \cdot \nabla \bar{T} d x
\end{gathered}
$$

and where $\bar{T}$ is the test function.

We define the general objective or constraint function of a linear thermal conduction represented as a functional $J(\phi)=\int_{\Omega} j(\phi, T) \mathrm{d} x$. The derivative of this function with respect to $\phi$ in the direction $\theta$ is:

$$
\begin{aligned}
\left\langle J^{\prime}(\phi), \theta\right\rangle & =\int j^{\prime}(\phi) \theta d x+\int j^{\prime}(\tilde{T})\left\langle\tilde{T}^{\prime}(\phi), \theta\right\rangle d x \\
& =\int j^{\prime}(\phi) \theta d x+\int j^{\prime}(\tilde{T}) \bar{T} d x
\end{aligned}
$$

Setting adjoint state $q$ as test function of the weak-form equations of state in Eq. (A.1), the Lagrangian is formulated as:

$$
L(\phi, \tilde{T}, q)=\int j(\phi, \tilde{T}) d x+a(\tilde{T}, q)+l(q)
$$

Using this expression, the derivative of the objective function can be expressed as:

$$
\begin{aligned}
\left\langle j^{\prime}(\phi), \theta\right\rangle & =\left\langle\frac{\partial L}{\partial \phi}(\phi, \tilde{T}, q), \theta\right\rangle+\left\langle\frac{\partial L}{\partial \tilde{T}}(\phi, \tilde{T}, q),\left\langle\tilde{T}^{\prime}(\phi), \theta\right\rangle\right\rangle \\
& =\left\langle\frac{\partial L}{\partial \phi}(\phi, \tilde{T}, q), \theta\right\rangle+\left\langle\frac{\partial L}{\partial \tilde{T}}(\phi, \tilde{T}, q), \bar{T}\right\rangle
\end{aligned}
$$


where $\bar{T}=\left\langle\tilde{T}^{\prime}(\phi), \theta\right\rangle$.

Consider the case where the second term is zero. This term is calculated as:

$$
\left\langle\frac{\partial L}{\partial \tilde{T}}, \bar{T}\right\rangle=\int j^{\prime}(\tilde{T}) \bar{T} d x+a(\bar{T}, q)=0
$$

When the adjoint state $q$ satisfies the above adjoint equation, the second term of Eq. (A.6) can be ignored. The derivatives of Eq. (A.1) with respect to $\phi$ in the direction $\theta$ are:

$$
d a(\tilde{T}, q)+a(\bar{T}, q)+d l(q)=0
$$

where

$$
\begin{gathered}
d a(\tilde{T}, q)=\int_{\Omega} \lambda^{\prime}(\phi) \nabla \tilde{T} \cdot \nabla q \theta d x \\
d l(q)=\int \lambda^{\prime}(\phi) \nabla T_{0} \cdot \nabla q \theta d x
\end{gathered}
$$

By substituting Eq. (A.8) into Eq. (A.7), it follows that:

$$
j^{\prime}(\tilde{T}) \bar{T} d x=d a(\tilde{T}, q)+d l(q)
$$

Substituting Eq. (A.11) into Eq. (A.4) yields:

$$
\begin{aligned}
J^{\prime}(\phi) & =j^{\prime}(\phi)+\lambda^{\prime}(\phi) \nabla \tilde{T} \cdot \nabla q+\lambda^{\prime}(\phi) \nabla T_{0} \cdot \nabla q \\
& =j^{\prime}(\phi)+\lambda^{\prime}(\phi) \nabla q \nabla\left(\tilde{T}+T_{0}\right)
\end{aligned}
$$

The effective thermal conductivity $\lambda_{(i i)}^{*}$ in the i-direction is first obtained as follows from Eq. (2):

$$
\lambda_{(i i)}^{*}=\int \lambda \nabla T \cdot \nabla T d x
$$


where $T$ is the solution of Eq. (1) under the Direclet boundary conditions and generates $\overline{\nabla T}_{i}=1$ and $\overline{\nabla T}_{j(\neq i)}=0$ on the unit volume $(V=1)$ domain. By substituting $j(\phi)=\lambda \nabla T \cdot \nabla$ into Eq. (A.7), the adjoint equation is obtained:

$$
2 a\left(\tilde{T}+T_{0}, \bar{T}\right)+a(q, \bar{T})=0
$$

Thus, $q=-2\left(\tilde{T}+T_{0}\right)$. Substituting $j(\phi)$ and $q$ into Eq. (A.12) yields the sensitivity:

$$
J^{\prime}(\phi)=-\lambda^{\prime}(\phi)\left(\tilde{T}+T_{0}\right) \nabla\left(\tilde{T}+T_{0}\right)=-\lambda^{\prime}(\phi) \nabla T \nabla T
$$

